\title{
Defects and Oxide Ion Migration in the Solid Oxide Fuel Cell Cathode Material $\mathrm{LaFeO}_{3}$
}

\author{
Felicity H. Taylor,* John Buckeridge, and C. Richard A. Catlow
}

Kathleen Lonsdale Materials Chemistry, Department of Chemistry, University College London, 20 Gordon Street, London, WC1H 0AJ, United Kingdom

\section{Supporting Information}

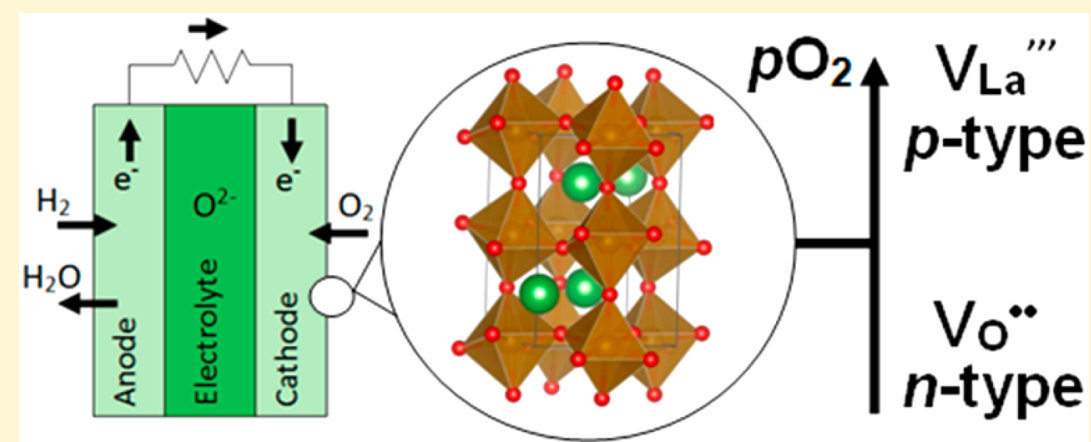

ABSTRACT: $\mathrm{LaFeO}_{3}$, a mixed ionic electronic conductor, is a promising cathode material for intermediate temperature solid oxide fuel cells (IT-SOFC). Key to understanding the electronic and ion conducting properties is the role of defects. In this study $\mathrm{ab}$ initio and static lattice methods have been employed to calculate formation energies of the full range of intrinsic defectsvacancies, interstitials, and antisite defects - under oxygen rich and oxygen poor conditions, to establish which, if any, are likely to occur and the effect these will have on the properties of the material. Under oxygen rich conditions, we find that the defect chemistry favors $p$-type conductivity, in excellent agreement with experiment, but contrary to previous studies, we find that cation vacancies play a crucial role. In oxygen poor conditions $\mathrm{O}^{2-}$ vacancies dominate, leading to $n$-type conductivity. Finally, static lattice methods and density functional theory were used to calculate activation energies of oxide ion migration through this material. Three pathways were investigated between the two inequivalent oxygen sites, $\mathrm{O} 1$ and $\mathrm{O} 2 ; \mathrm{O} 2-\mathrm{O} 2, \mathrm{O} 1-\mathrm{O} 2$, and $\mathrm{O} 1-$ $\mathrm{O} 1$, with $\mathrm{O} 2-\mathrm{O} 2$ giving the lowest activation energy of $0.58 \mathrm{eV}$, agreeing well with experimental results and previous computational studies.

\section{INTRODUCTION}

With the issues of environmental impact and security surrounding energy generation, the need for a clean and renewable alternative is becoming increasingly essential. Fuel cells are able to convert fuel directly to electricity, without a combustion step, making them more efficient than combustion engines, as well as producing less emissions and noise pollution. Different types of fuel cells have been investigated, including polymer electrolyte membrane ${ }^{1}$ and proton exchange membrane fuel cells. ${ }^{2}$ However, solid oxide fuel cells (SOFCs) offer many advantages over these as they are the cleanest and most efficient: ${ }^{3}$ they alleviate the need for corrosive liquids and have been shown to be reliable when operated continuously. ${ }^{1}$ The efficiency of these fuel cells depends on their component materials, thus finding optimal materials is an active field of research.

Fuel cells have three main components: the cathode, electrolyte, and anode. An oxidant, typically air, is reduced at the cathode producing $\mathrm{O}^{2-}$ ions which migrate across the electrolyte to the anode where they react with a fuel, usually hydrogen. The flow of ionic charge from cathode to anode is balanced by the flow of electrons, from anode to cathode, through an external circuit. ${ }^{1,4}$ This conduction of ions is a thermally activated process, leading to high operating temperatures which in turn cause short lifetimes and the need for expensive materials for each of the component parts. Therefore, research into SOFCs has focused on reducing the temperature of operation to between 500 and $800{ }^{\circ} \mathrm{C}$.

In order to achieve such a change in fuel cell technology, novel materials, active at reduced temperatures, are required, particularly for the cathode, the site of the oxygen reduction reaction (ORR). The ORR becomes the limiting step of the fuel cell at low temperatures due to its high activation energy and the limited area within which this reaction can take place in conventional cathodes: at the triple phase boundary (TPB), where the electrode, electrolyte, and electrochemical gas meet. ${ }^{4}$

Mixed ionic and electronic conductors, such as perovskite $\mathrm{LaFeO}_{3}$, show strong potential as cathode materials as, in

Received: July 25, 2016

Revised: October 9, 2016

Published: November 2, 2016 
addition to the electronic conductivity required to transport electrons to the reaction site, the ionic conductivity serves to increase the area within which the oxygen reduction reaction can take place. Both these properties can be improved with doping on the A- and B-sites, leading to double doped systems, e.g., $\mathrm{La}_{1-x} \mathrm{Sr}_{x} \mathrm{Co}_{1-y} \mathrm{Fe}_{y} \mathrm{O}_{3-\delta}$ (LSCF), which have shown high electronic and ionic conductivities along with high catalytic activity and low thermal expansion coefficients, making them more compatible with common electrolytes. ${ }^{5-9}$

However, in order to optimize fully $\mathrm{LaFeO}_{3}$ as a SOFC cathode, it is first necessary to gain an in depth knowledge of the defects present in this system, their likely concentrations under operating conditions, and their effects on the properties of the material. Determining the precise role of point defects using experimental techniques is highly challenging, making computational techniques invaluable in this respect. In this study, we have therefore investigated point defects in $\mathrm{LaFeO}_{3}$ and determined their formation energies using density functional theory (DFT).

Defects in $\mathrm{LaFeO}_{3}$ have been investigated previously using a range of computational approaches, including interatomic potential based methods ${ }^{10-12}$ and DFT. ${ }^{13}$ Jones and Islam, ${ }^{10}$ using interatomic potential methods, found Schottky and Frenkel disorder high energy, suggesting electronic conductivity is only possible in acceptor-doped $\mathrm{LaFeO}_{3}$, while Ritzmann et al. ${ }^{13,14}$ have used DFT+U to studied oxygen vacancy formation in $\mathrm{LaFeO}_{3}$ and found a high enthalpy of oxygen vacancy formation leading to a low concentration of oxygen vacancies in the stoichiometric material. Defect models, based on experimental data, have been proposed by Mizusaki et al. ${ }^{15}$ and Wærnhus et al., ${ }^{16,17}$ and while Mizusaki et al. ${ }^{15}$ propose the electronic properties of $\mathrm{LaFeO}_{3}$ at high oxygen partial pressures were governed by lanthanum vacancies, Wærnhus et al. ${ }^{16,17}$ suggest that both lanthanum and iron vacancies are involved. Although the effect of lanthanum vacancies on oxygen vacancy concentration has been investigated by Ritzmann et al., ${ }^{13}$ their mode of incorporation into the system is yet to be studied, and the role of iron vacancies has not been addressed.

In this paper, intrinsic point defects in $\mathrm{LaFeO}_{3}$ have been investigated using DFT and interatomic potential based methods. Formation energies of vacancies, interstitials, and antisite defects have been calculated under different growth environments, with $\mathrm{La}^{3+}$ and $\mathrm{Fe}^{3+}$ vacancies, formed through Schottky disorder, dominating under oxygen rich conditions, whereas $\mathrm{O}^{2-}$ vacancies dominate under oxygen poor conditions. These results explain the conductivity regimes observed in this material under varying oxygen partial pressure. Finally, the activation energies of oxide ion migration have been calculated for the different pathways present in $\mathrm{LaFeO}_{3}$, which allow the lowest energy pathway to be identified.

\section{SIMULATION METHOD}

At low and ambient temperatures, the structure of $\mathrm{LaFeO}_{3}$ is the orthorhombic perovskite form, Pbnm No. 62, ${ }^{18}$ shown in Figure 1, which has been used throughout our investigation. In our study, we have determined formation energies of intrinsic defects using two different methods: static lattice and ab initio. The approaches used for both methods are outlined below.

Static Lattice. Static lattice calculations are based on the Born model of a solid, comprising a long-range Coulombic interaction between each pair of ions and a short-range Buckingham term which models the overlap repulsion and van der Waals forces in the form:

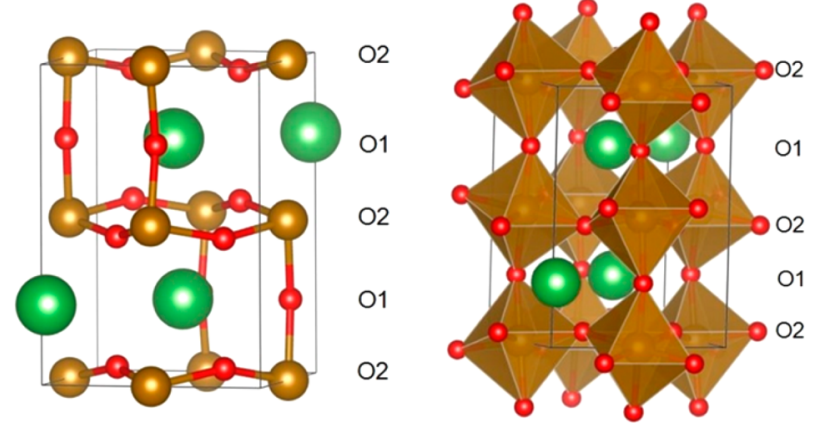

Figure 1. Orthorhombic form of $\mathrm{LaFeO}_{3}$; lanthanum is shown in green, iron in gold, and oxygen in red. Labels $\mathrm{O} 1$ and $\mathrm{O} 2$, to the right of the structure, show the two oxygen environments.

$$
V_{i j}(r)=A_{i j} \mathrm{e}^{\left(-r_{i j} / \rho_{i j}\right)}-\frac{c_{i j}}{r_{i j}^{6}}
$$

where $V_{i j}$ is the short-range potential energy between the ions $i$ and $j$, and $A_{i j}, \rho_{i j}$, and $C_{i j}$ are the potential parameters particular to each pair. The electronic polarizability of the ions in $\mathrm{LaFeO}_{3}$ is described using the shell model, in which the ion is represented as a core connected to a charge-bearing shell by a harmonic spring. ${ }^{19}$ The short-range potential parameters used in the calculations of defect energies were derived by Cherry et al. ${ }^{12}$ by empirical fitting to observed structural properties of cubic perovskites, using a cutoff of $12 \AA$ for all potentials. These potential parameters reproduced the orthorhombic structure of $\mathrm{LaFeO}_{3}$ to within $0.55 \%$ of the experimental lattice parameters, as shown in Table 1.

Table 1. Calculated and Experimental ${ }^{23}$ Lattice Parameters for Orthorhombic $\mathrm{LaFeO}_{3}$, Calculated Using Interatomic Potentials

\begin{tabular}{cccc} 
parameter & calculated & experimental & \% difference \\
$a, \AA$ & 5.558 & 5.553 & 0.090 \\
$b, \AA$ & 5.564 & 5.563 & 0.018 \\
$c, \AA$ & 7.905 & 7.862 & 0.547 \\
\hline
\end{tabular}

The Mott-Littleton methodology ${ }^{20}$ was implemented for the calculations of defect formation energy and ion migration. For each system studied the radius of region one was increased until the calculated defect energy reached convergence, below $0.003 \mathrm{eV}$. The radius of region one therefore varies, depending on the calculation, between the values of 7.0 and $12.0 \AA$, which corresponds to between 266 and 1222 ions.

For the ion migration calculations, five points, at equal intervals, were chosen along a straight line connecting two oxygen sites within the material. Previous ion migration studies on perovskite-type oxides have shown that the ion can travel via a curved path., 21 Therefore, at each chosen position along the straight line, the energy of the migrating oxygen species was calculated on a two-dimensional grid around the original position, in a perpendicular direction to the oxide ion path, in order to find the lowest energy point for the oxide ion. Each energy point was calculated using the Mott-Littleton methodology to relax the crystal structure, in region one, around the fixed migrating oxide ion. It was assumed that the saddle point of the migration path would be situated between the two highest energy points along the path. Therefore, the halfway point between these two points was calculated, and the saddle 
Table 2. Lattice Parameters, Bond Lengths, Angles, Relaxed Cell Volume, Band Gap, and Magnetic $\mathrm{Moment}_{\text {of }} \mathrm{LaFeO}_{3}$ with Increasing $U_{\text {eff }}$ Using GGA $+U$, along with Experimental Values

\begin{tabular}{|c|c|c|c|c|c|c|}
\hline \multirow[b]{2}{*}{ parameters } & \multirow[b]{2}{*}{$\exp$} & \multicolumn{5}{|c|}{$U$} \\
\hline & & 4 & 5 & 6 & 7 & 8 \\
\hline$a(\AA)$ & $5.553^{a}$ & 5.572 & 5.571 & 5.567 & 5.563 & 5.558 \\
\hline$b(\AA)$ & $5.563^{a}$ & 5.638 & 5.629 & 5.620 & 5.608 & 5.588 \\
\hline$c(\AA)$ & $7.862^{a}$ & 7.903 & 7.902 & 7.898 & 7.887 & 7.875 \\
\hline $\mathrm{Fe}-\mathrm{O}_{\mathrm{ax}}(\AA)$ & $2.009^{a}$ & 2.028 & 2.027 & 2.025 & 2.020 & 2.016 \\
\hline $\mathrm{Fe}-\mathrm{O}_{\mathrm{eq}}(\AA)$ & $2.007^{a}$ & 2.039 & 2.035 & 2.031 & 2.026 & 2.018 \\
\hline $\mathrm{Fe}-\mathrm{O}_{\mathrm{eq}}(\AA)$ & $2.002^{a}$ & 2.027 & 2.024 & 2.020 & 2.016 & 2.008 \\
\hline $\mathrm{La}-\mathrm{O}(\AA)$ & $2.416^{a}$ & 2.409 & 2.411 & 2.410 & 2.413 & 2.410 \\
\hline $\mathrm{La}-\mathrm{O}(\AA)$ & $2.591^{a}$ & 2.539 & 2.545 & 2.550 & 2.554 & 2.572 \\
\hline $\mathrm{La}-\mathrm{O}(\AA)$ & $2.455^{a}$ & 2.443 & 2.443 & 2.444 & 2.446 & 2.449 \\
\hline $\mathrm{La}-\mathrm{O}(\AA)$ & $2.656^{a}$ & 2.662 & 2.663 & 2.662 & 2.661 & 2.650 \\
\hline $\mathrm{La}-\mathrm{O}(\AA)$ & $2.805^{a}$ & 2.796 & 2.798 & 2.799 & 2.796 & 2.809 \\
\hline $\mathrm{Fe}-\mathrm{O}-\mathrm{Fe}_{\mathrm{ax}}(\mathrm{deg})$ & $156.32^{a}$ & 153.832 & 154.126 & 154.385 & 154.818 & 155.138 \\
\hline $\mathrm{Fe}-\mathrm{O}-\mathrm{Fe}_{\mathrm{eq}}(\mathrm{deg})$ & $157.22^{a}$ & 154.296 & 154.620 & 154.967 & 155.398 & 156.306 \\
\hline volume of cell $\left(\AA^{3}\right)$ & $58.053^{b}$ & 62.065 & 61.950 & 61.775 & 61.520 & 61.148 \\
\hline band gap $(\mathrm{eV})$ & $2.1^{c}$ & 1.681 & 2.031 & 2.329 & 2.626 & 2.924 \\
\hline magnetic moment $\left(\mu_{\mathrm{B}}\right)$ & $4.6 \pm 0.2^{d}$ & \pm 4.14 & \pm 4.22 & \pm 4.31 & \pm 4.40 & \pm 4.51 \\
\hline
\end{tabular}

point was found using the rational function optimization (RFO) method of minimization allowing the migrating ion to relax as well as the crystal structure. The center of the defect calculation was kept constant throughout each ion migration calculation, as the middle point between the two oxygen vacancy sites, thus keeping systematic errors to a minimum. The energy at the saddle point, along with the lowest oxygen vacancy formation energy, was then used to calculate the activation energy. All static lattice calculations were carried out using the GULP 22 code.

Ab Initio. All first principle, electronic structure calculations were performed using spin-polarized Kohn-Sham DFT ${ }^{24,25}$ and DFT $+U^{26}$ using the plane-wave pseudopotential technique with the projector augmented-wave ${ }^{27}$ (PAW) approach to model the core-valence electron interaction, as implemented in the Vienna ab initio simulation package (VASP). ${ }^{28-30}$ The valence configurations used were $\mathrm{La}\left(5 \mathrm{~s}^{2} 5 \mathrm{p}^{6} 6 \mathrm{~s}^{2} 5 \mathrm{~d}^{1}\right), \mathrm{Fe}\left(4 \mathrm{~s}^{2}\right.$ $\left.3 \mathrm{~d}^{8}\right)$, O $\left(2 \mathrm{~s}^{2} 2 \mathrm{p}^{4}\right)$. The electron exchange and correlation were evaluated within the generalized gradient approximation (GGA $+U)$ using the functional of Perdew, Burke, and Ernzerhof (PBE), ${ }^{31}$ which was found to reproduce the experimental structure of $\mathrm{LaFeO}_{3}$ most accurately, after comparison with the local density approximation $(\mathrm{LDA}+U)$, and $\mathrm{GGA}+U$ with the Perdew, Berke, and Ernzerhof functional revised for solids (PBEsol); ${ }^{32}$ see Supporting Information. A k-mesh of $(4 \times 4 \times$ 3) generated by the Monkhorst-Pack ${ }^{33}$ scheme is used for Brillouin zone sampling for the $\mathrm{LaFeO}_{3}$ unit cell. Integration over the first Brillouin zone used Gaussian smearing $(\sigma=0.05)$ during structural relaxations. The planewave basis set was converged at a kinetic cutoff of $650 \mathrm{eV}$, with the total energy converging to within $0.001 \mathrm{eV}$ per atom.

A $2 \times 2 \times 1$ supercell with antiferromagnetic $\mathrm{G}$ ordering of the $\mathrm{Fe}^{3+}$ ions was used for all defect calculations. The energies of supercells containing defects were calculated at the lattice parameters of the relaxed perfect supercell. When investigating interstitials an initial interstitial position at the center of the $2 \times$ $2 \times 1$ supercell was used.

A $U_{\text {eff }}$ parameter, which represents the difference between the Coulomb $(U)$ and exchange $(J)$ parameters $\left(U_{\text {eff }}=U-J\right)$, is utilized in the DFT $+U$ calculations, in order to correct for the inadequate description of localized $3 \mathrm{~d}$ electrons on transition metals. ${ }^{26}$ A value of $7.0 \mathrm{eV}$ was used for $\mathrm{Fe}^{3+}$, determined after comparing experimental structure parameters and properties $^{23,34-37}$ to those calculated at increasing values of $U_{\text {eff, }}$ the results of which can be seen in Table 2 . This value is higher than previously reported $U_{\text {eff }}$ values for $\mathrm{Fe}^{3+}$ in $\mathrm{LaFeO}_{3}$, which include $4.3,^{13,15} 5.1,^{38}$ and $5.4 \mathrm{eV} ;{ }^{39}$ however, $U_{\text {eff }}=7.0 \mathrm{eV}$ provides a balance between optimally reproducing the structural parameters, which improve with increasing $U_{\text {eff }}$ value, and the band gap, which agrees best with experiment at $U_{\text {eff }}=5.0 \mathrm{eV}$. A value of $7.0 \mathrm{eV}$ also reproduces the experimental magnetic moment for $\mathrm{Fe}^{3+}$ whereas lower values do not; therefore, it is the most appropriate $U_{\text {eff }}$ value to employ. The use of hybrid functionals for exchange and correlation in $\mathrm{LaFeO}_{3}$, which are significantly more computationally demanding than standard DFT, does not offer clear advantages in terms of crystal, electronic, and magnetic structure $^{40}$ over GGA $+U$ and hence was not considered in this study.

Calculation of Defect Formation Energies Using DFT $+U$. Formation energies for defects, in charge state $q\left(\Delta H_{\mathrm{f}}(q)\right)$, were calculated using the following equation:

$$
\begin{aligned}
\Delta H_{\mathrm{f}}(q)= & E^{\text {defect }}-E^{\text {perfect }} \pm \sum n_{x}\left(E_{x}+\mu_{x}\right) \\
& +q\left(E_{\mathrm{VBM}}+E_{\mathrm{F}}+\Delta E_{\mathrm{pot}}\right)+q^{2} E_{\mathrm{ic}}
\end{aligned}
$$

with $E^{\text {defect }}$ being the cohesive energy of the relaxed supercell containing the defect, $E^{\text {perfect }}$ the energy of the relaxed perfect supercell, $n_{x}$ the number of defect atoms or ions added or removed when forming the defect, $E_{x}$ the elemental reference energy, i.e., the energy of an element in its standard state, and $\mu_{x}$ the chemical potential of the defect, where $x$ is $\mathrm{La}, \mathrm{Fe}$, or $\mathrm{O}$. The elemental reference energies of lanthanum and iron were calculated using the respective metals and oxygen by using a triplet oxygen molecule in a large supercell. For charged defects, where $q \neq 0$, the chemical potential of the electrons in the system needs to be taken into account, which is done with $E_{\mathrm{VBM}}$, the energy of the valence band maximum (VBM), and $E_{\mathrm{F}}$, the Fermi energy, defined with respect to the VBM. In addition, 
a correction term is required, comprising $\Delta E_{\text {pot }}$ the correction that accounts for the difference between the potential of the perfect supercell and the supercell containing the charged defect, and $E_{\mathrm{ic}}$, the correction to the interaction of charged defects with their periodic image. ${ }^{41}$

Two methods of calculating $E_{\mathrm{ic}}$ were investigated: one designed for cubic systems, in which the dielectric tensor is constant along $a, b$, and $c$, ${ }^{41}$ and the other which takes into account the anisotropy between dielectric tensors along different lattice parameters. ${ }^{42}$ Orthorhombic $\mathrm{LaFeO}_{3}$ is only slightly distorted from the cubic perovskite structure and therefore only small differences, reaching a maximum of 0.026 $\mathrm{eV}$ for $q= \pm 3$, were found between the correction values calculated by each method, demonstrating that the choice of method has minimal impact on our results. However, in order to model defects in orthorhombic $\mathrm{LaFeO}_{3}$ as accurately as possible, the method outlined by Murphy and Hine, ${ }^{42}$ which takes into account the differences in dielectric tensors along the $a, b$, and $c$ lattice parameters, was selected.

The formation energy of defects under different conditions can be investigated by varying the chemical potential, $\mu_{x}$, in eq 2 , which requires the range of chemical potentials under which $\mathrm{LaFeO}_{3}$ forms preferentially, over competing phases, to be calculated. We use the chemical potential limits analysis program (CPLAP), ${ }^{43}$ which employs calculated enthalpies of formation, at thermodynamic equilibrium, of $\mathrm{LaFeO}_{3}$ and its competing phases, to determine the ranges of chemical potentials in which $\mathrm{LaFeO}_{3}$ is stable with respect to these phases. The result is shown as the gray shaded region in Figure 2.

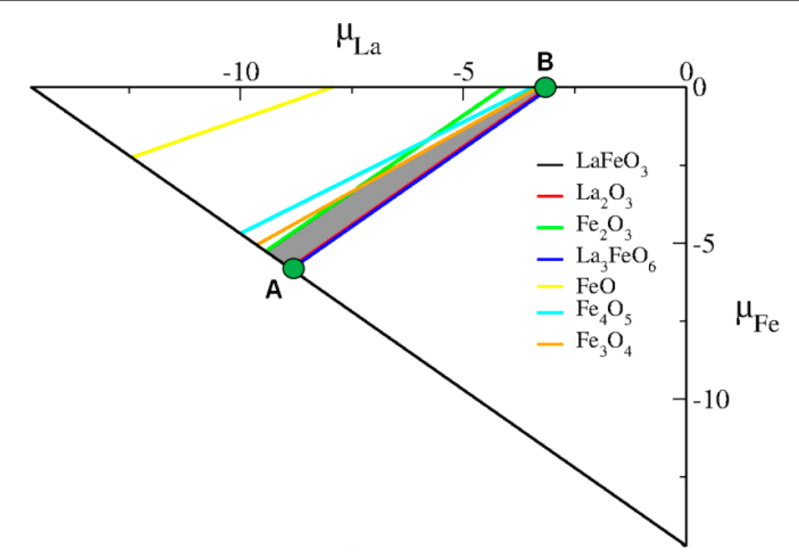

Figure 2. Accessible chemical potential ranges for $\mathrm{LaFeO}_{3}$ with respect to competing phases. The shaded region indicates the chemical potential values where $\mathrm{LaFeO}_{3}$ is stable.

Defect formation energies were calculated at the chemical potential values of two different points within this shaded region, marked $\mathrm{A}$ and $\mathrm{B}$. These points were chosen as they represent the extremes of possible conditions in which $\mathrm{LaFeO}_{3}$ forms preferentially: A, bound by $\mathrm{La}_{2} \mathrm{O}_{3}$ and $\mathrm{LaFeO}_{3}$, represents lanthanum and iron poor and oxygen rich conditions, with chemical potential values $\mu_{\mathrm{La}}=-8.89 \mathrm{eV}$, $\mu_{\mathrm{Fe}}=-5.80 \mathrm{eV}$, and $\mu_{\mathrm{O}}=0.00 \mathrm{eV}$, and $\mathrm{B}$, bound by $\mathrm{La}_{2} \mathrm{O}_{3}$ and Fe metal, represents lanthanum and iron rich and oxygen poor conditions, with chemical potential values $\mu_{\mathrm{La}}=-3.09 \mathrm{eV}, \mu_{\mathrm{Fe}}=$ $0.00 \mathrm{eV}$, and $\mu_{\mathrm{O}}=-3.87 \mathrm{eV}$. Oxygen rich conditions represent both operating and growth conditions of SOFC cathode materials whereas oxygen poor conditions represent reducing conditions; the use of these two regimes allows us to investigate how varying oxygen partial pressure affects this material and its defect chemistry.

Calculating Oxygen Ion Migration Barriers Using DFT $+U$. Following on from the static lattice ion migration calculation, the activation energies were also calculated using $\mathrm{DFT}+U$. As with the defect formation energies, the GGA functional was utilized with a $U_{\text {eff }}$ value of $7.0 \mathrm{eV}$ used for the $\mathrm{Fe}^{3+}$ ions. The position of the oxide ion at the saddle point of each pathway was taken from the static lattice calculations and used to position the oxide ion in our 80 atom supercell. All other ions in the supercell were then relaxed, keeping the migrating ion fixed. The formation energies of oxygen vacancies at the beginning and end of the migration pathway were calculated, and the activation energy was evaluated as the difference between the supercell with the saddle point oxide ion and the supercell containing the oxygen vacancy. As with our employment of the Mott-Littleton approach (see above), where the energies of the oxygen vacancies at the beginning and end of the migrating pathway differed, the lowest energy oxygen vacancy was used.

\section{RESULTS AND DISCUSSION}

Intrinsic Defects. The formation energies of intrinsic defects, both neutral and charged, within the two chemical potential environments, have been calculated using the DFT technique. Table 3 , in which standard Kröger-Vink notation ${ }^{44}$ is

Table 3. Defect Formation Energies of Neutral Vacancies in $\mathrm{LaFeO}_{3}$

\begin{tabular}{lcc} 
& \multicolumn{2}{c}{ defect formation energy $(\mathrm{eV})$} \\
\cline { 2 - 3 } neutral defect & $\mathrm{O}$ Rich/La and Fe Poor & $\mathrm{O}$ Poor/La and Fe Rich \\
$\mathrm{V}_{\mathrm{La}}^{x}$ & 3.333 & 9.136 \\
$\mathrm{~V}_{\mathrm{Fe}}^{x}$ (spin up) & 2.639 & 8.442 \\
$\mathrm{~V}_{\mathrm{Fe}}^{x}$ (spin down) & 2.940 & 8.743 \\
$\mathrm{~V}_{\mathrm{O}}^{x}(\mathrm{O} 1)$ & 3.960 & 0.092 \\
$\mathrm{~V}_{\mathrm{O}}^{x}(\mathrm{O} 2)$ & 3.829 & -0.040 \\
\hline
\end{tabular}

used, shows the defect energies of neutral vacancies of $\mathrm{La}, \mathrm{Fe}$, and $\mathrm{O}$. Due to the antiferromagnetic ordering of iron ions in $\mathrm{LaFeO}_{3}$, iron in spin up and spin down orientations, with respect to the $c$ lattice parameter, is present (as can be seen in Figure 3), and therefore defects involving iron in each orientation have been investigated.

Two values are also reported for oxygen defects as there are two inequivalent oxygen sites in $\mathrm{LaFeO}_{3}, \mathrm{O} 1$ and $\mathrm{O}$, corresponding to axial and equatorial positions in the $\mathrm{Fe}$ centered octahedra; defects involving both sites have been calculated throughout the study.

The values in Table 3 are high in most cases and indicate that neutral vacancies will not be present in significant concentrations in stoichiometric $\mathrm{LaFeO}_{3}$, with the exception of neutral oxygen vacancies, particularly $\mathrm{O} 2$ oxygen vacancies under oxygen poor conditions; a key result as oxygen vacancies are necessary for oxygen transport through this material. As expected, vacancy formation energies for all species are lower when in "poor conditions" for that species.

The formation energies of oxygen vacancies have been calculated previously by Mastrikov et al. ${ }^{45}$ and then by Ritzmann et al. ${ }^{14}$ who reported values of 4.41 and $4.01 \mathrm{eV}$, respectively, comparing well with the values calculated here, and 3.960 and $3.829 \mathrm{eV}$ for oxygen rich conditions. The 


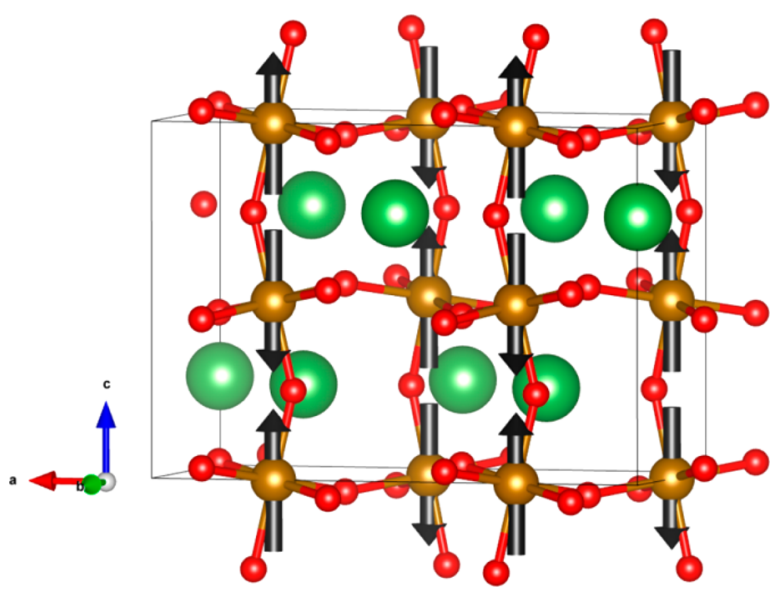

Figure 3. Supercell $(2 \times 1 \times 1)$ of $\mathrm{LaFeO}_{3}$ showing the spin orientations on iron; the black arrows represent the magnetic moment of the iron ion, either +4.4 or $-4.4 \mu \mathrm{B}$.

negative formation energy for oxygen vacancies under oxygen poor conditions suggests that this material will be significantly oxygen deficient under reducing atmospheres.

Comparing the formation energies of neutral vacancies to those of neutral interstitials, shown in Table 4, in general interstitials have higher formation energies, as is to be expected for a close packed material such as $\mathrm{LaFeO}_{3}$.

Table 4. Defect Formation Energies of Neutral Interstitials in $\mathrm{LaFeO}_{3}$

\begin{tabular}{lcc} 
& \multicolumn{2}{c}{ defect formation energy $(\mathrm{eV})$} \\
\cline { 2 - 3 } neutral defect & $\mathrm{O}$ Rich/La and Fe Poor & O Poor/La and Fe Rich \\
$\mathrm{La}_{i}^{x}$ & 12.550 & 6.747 \\
$\mathrm{Fe}_{i}^{x}$ (spin up) & 8.708 & 2.904 \\
$\mathrm{Fe}_{i}^{x}$ (spin down) & 8.006 & 2.203 \\
$\mathrm{O}_{i}^{x}$ & 1.628 & 5.496 \\
\hline
\end{tabular}

Initially, interstitials were placed at the center of the $2 \times 2 \times$ 1 supercell, occupying square planar positions between four oxygens: position $0.5,0.5,0.5$ in fractional coordinates. However, examination of the optimized position of lanthanum and oxygen interstitials reveals that they form split interstitials with a nearby ion on its lattice site, as can be seen in Figure 4a,b. Split interstitials have been proposed in tightly packed structures such as perovskites, ${ }^{46,47}$ and given that single interstitials of $\mathrm{La}$ and $\mathrm{O}$ could not be stabilized, these results suggest that split interstitials are more favorable than single interstitials for both lanthanum and oxygen. The preferential

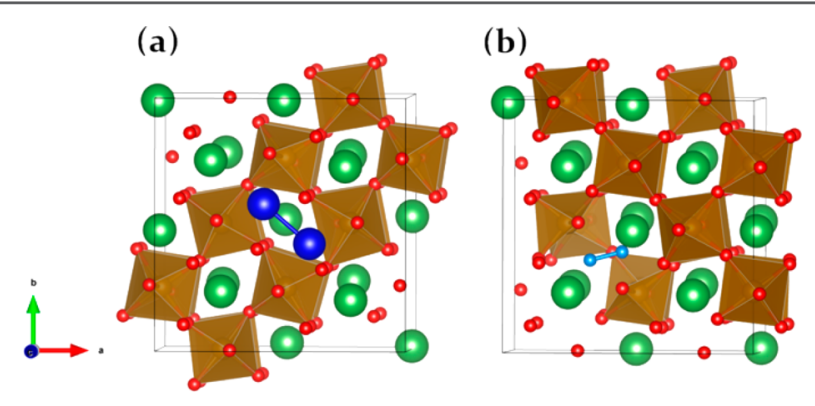

Figure 4. Split interstitials of (a) lanthanum, shown in dark blue, and (b) oxygen, shown in pale blue. formation of split interstitials for the A-site and oxygen ions has been found in similar materials including the $(\mathrm{Ba}, \mathrm{Sr})$ $(\mathrm{Co}, \mathrm{Fe}) \mathrm{O}_{3-\delta}$ (BSCF) family of perovskites. ${ }^{48,49}$

There are a number of different orientations for split interstitials within this structure, which were investigated to find the most favorable structure. For both lanthanum and oxygen, splitting the interstitial along the three lattice parameters, $a, b$, and $c$, was investigated, with configurations along and perpendicular to the $\mathrm{Fe}-\mathrm{O}-\mathrm{Fe}$ bond also investigated for oxygen.

It was found that the orientations shown in Figure 4a,b are the most stable for lanthanum and oxygen split interstitials; $(-a, 0.84 b)$ for lanthanum and $(-a,-0.26 b, 0.11 c)$ for oxygen. Iron split interstitials were also investigated. However, all orientations studied converged into single interstitials, suggesting that this is the most favorable form for iron. These conclusions match with those drawn from our interatomic potential calculations, which found lanthanum and oxygen split interstitials more stable than single interstitials, whereas single interstitials were more stable for iron, as shown in Table 5.

Table 5. Defect Energies (eV) of Single and Split Interstitials in $\mathrm{LaFeO}_{3}$ Calculated Using Interatomic Potentials

$\begin{array}{lcc}\text { defect } & \text { single } & \text { split } \\ \mathrm{La}_{i} \cdots & -23.73 & -25.73 \\ \mathrm{Fe}_{i} \cdots & -39.38 & -38.74 \\ \mathrm{O}_{i}{ }^{\prime \prime} & -10.99 & -11.78\end{array}$

The optimized position of iron interstitials, in both orientations, is shown in Figure 5b. Figure 5a shows the initial
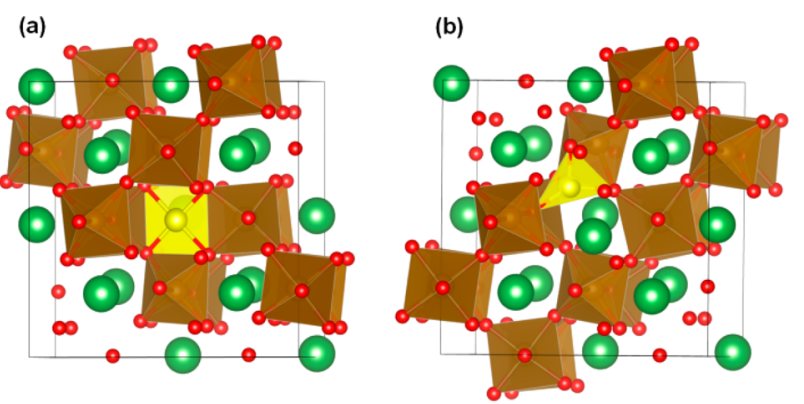

Figure 5. Change in position of iron interstitial found in this material. The iron interstitial is shown in yellow: (a) between two lanthanum ions, the front lanthanum ion has been removed from the image for ease of viewing. This is the position initially used for all interstitials; it has a square planar configuration with its surrounding oxygen ions (b) displaced from this site in $a-a, b$ direction; it has a tetrahedral configuration with its surrounding oxygen ions.

position of the interstitial, which has a square planar configuration. In Figure $5 b$ we see that the interstitial has shifted to a tetrahedral position. The magnetic moment of the interstitial in the tetrahedral position is $3.7 \mu_{\mathrm{B}}$, a decrease from the bulk magnetic moment of $4.4 \mu_{\mathrm{B}}$, suggesting that iron interstitials are incorporated as $\mathrm{Fe}^{2+}$ ions, with the excess charge delocalized onto the oxygens surrounding the interstitial.

Frenkel and Schottky Type Disorder. The formation energies calculated for vacancies and interstitials of lanthanum, iron, and oxygen can be used to calculate the energies of Frenkel and Schottky type disorder, which are represented as follows. 
Frenkel disorder:

$$
\mathrm{A}_{\mathrm{A}}^{x} \leftrightarrow \mathrm{V}_{\mathrm{A}}^{x}+\mathrm{A}_{i}^{x}
$$

$\mathrm{La}_{2} \mathrm{O}_{3}$ partial Schottky disorder:

$$
2 \mathrm{La}_{\mathrm{La}}^{x}+3 \mathrm{O}_{\mathrm{O}}^{x} \leftrightarrow 2 \mathrm{~V}_{\mathrm{La}}^{x}+3 \mathrm{~V}_{\mathrm{O}}^{x}+\mathrm{La}_{2} \mathrm{O}_{3}
$$

$\mathrm{Fe}_{2} \mathrm{O}_{3}$ partial Schottky disorder:

$$
2 \mathrm{Fe}_{\mathrm{Fe}}^{x}+3 \mathrm{O}_{\mathrm{O}}^{x} \leftrightarrow 2 \mathrm{~V}_{\mathrm{Fe}}^{x}+3 \mathrm{~V}_{\mathrm{O}}^{x}+\mathrm{Fe}_{2} \mathrm{O}_{3}
$$

Full Schottky disorder:

$$
\mathrm{La}_{\mathrm{La}}^{x}+\mathrm{Fe}_{\mathrm{Fe}}^{x}+3 \mathrm{O}_{\mathrm{O}}^{x} \leftrightarrow \mathrm{V}_{\mathrm{La}}^{x}+\mathrm{V}_{\mathrm{Fe}}^{x}+3 \mathrm{~V}_{\mathrm{O}}^{x}+\mathrm{LaFeO}_{3}
$$

The energies of these processes have been calculated using DFT for both neutral and charged vacancies and interstitials, with the values calculated for Frenkel and Schottky disorder shown in Tables 6 and 7, respectively. The energies reported

Table 6. Energies of Frenkel Type Disorder in $\mathrm{LaFeO}_{3}$

\begin{tabular}{lcc} 
& \multicolumn{2}{c}{ energy per defect, eV } \\
\cline { 2 - 3 } Frenkel & neutral & charged \\
$\mathrm{La}$ & 7.941 & 5.807 \\
$\mathrm{Fe}(\mathrm{sd})$ & 5.473 & 4.444 \\
$\mathrm{Fe}(\mathrm{su})$ & 5.673 & 4.435 \\
$\mathrm{O} 1$ & 2.794 & 3.291 \\
$\mathrm{O} 2$ & 2.728 & 3.287 \\
\hline
\end{tabular}

Table 7. Energies of Schottky Disorder in $\mathrm{LaFeO}_{3}$

\begin{tabular}{lcc} 
& \multicolumn{2}{c}{ energy per defect, eV } \\
\cline { 2 - 3 } Schottky & neutral & charged \\
$\mathrm{La}_{2} \mathrm{O}_{3}$ partial O1 & 3.710 & 2.198 \\
$\mathrm{La}_{2} \mathrm{O}_{3}$ partial O2 & 3.631 & 2.193 \\
$\mathrm{Fe}_{2} \mathrm{O}_{3}$ partial O1 (sd) & 3.749 & 2.419 \\
$\mathrm{Fe}_{2} \mathrm{O}_{3}$ partial O2 (sd) & 3.670 & 2.414 \\
$\mathrm{Fe}_{2} \mathrm{O}_{3}$ partial O1 (su) & 3.629 & 2.414 \\
$\mathrm{Fe}_{2} \mathrm{O}_{3}$ partial O2 (su) & 3.550 & 2.409 \\
full O1 (sd) & 3.631 & 2.210 \\
full O2 (sd) & 3.552 & 2.205 \\
full O1 (su) & 3.571 & 2.207 \\
full O2 (su) & 3.492 & 2.203 \\
\hline
\end{tabular}

for Frenkel and Schottky disorder using charged defects have used defect energies for the $3+$ charge states for lanthanum and iron and $2-$ for oxygen. (The formation energies of Frenkel and Schottky disorder are independent of iron rich and poor conditions). The energies of Frenkel disorder using other charge states have been calculated and are reported in Table 8.

Table 8. Energies of Frenkel Type Disorder for Defects of Non-Zero Charge States

\begin{tabular}{lclc}
\multicolumn{1}{c}{ Frenkel } & energy defect & \\
$\mathrm{La}^{+}$ & $(\mathrm{eV})$ & \multicolumn{1}{c}{ Frenkel } & energy defect \\
$\mathrm{La}^{2+}$ & 6.956 & $\mathrm{Fe}^{2+}(\mathrm{su})$ & 4.197 \\
$\mathrm{La}^{3+}$ & 6.621 & $\mathrm{Fe}^{3+}(\mathrm{su})$ & 4.435 \\
$\mathrm{Fe}^{+}(\mathrm{sd})$ & 5.807 & $\mathrm{O}^{-}$ & 3.601 \\
$\mathrm{Fe}^{2+}(\mathrm{sd})$ & 4.778 & $\mathrm{O}^{2-}$ & 3.291 \\
$\mathrm{Fe}^{3+}(\mathrm{sd})$ & 4.287 & $\mathrm{O}^{-}$ & 3.615 \\
$\mathrm{Fe}^{+}(\mathrm{su})$ & 4.444 & $\mathrm{O}^{2-}$ & 3.287 \\
& 4.527 & &
\end{tabular}

For lanthanum and iron, charged Frenkel pairs have lower formation energies than their neutral counterparts; for oxygen, however, neutral Frenkel disorder will form preferentially. For Schottky disorder, $\mathrm{La}_{2} \mathrm{O}_{3}$ partial Schottky and full Schottky will form preferentially over $\mathrm{Fe}_{2} \mathrm{O}_{3}$ partial Schottky disorder.

The favorable formation of Schottky disorder supports work done by Mizusaki et al. $^{15}$ and the more recent work by Wærnhus et al., ${ }^{16,17}$ on electronic conductivity in $\mathrm{LaFeO}_{3}$ under varying $\mathrm{O}_{2}$ partial pressure. Both observed $p$-type conductivity at high partial pressures, with fully ionized cation vacancies required in order to keep the material charge neutral under this conductivity scheme. Mizusaki et al. assumed that lanthanum vacancies would be the most favorable due to the fixed stoichiometry of the iron with its surrounding oxygens, ${ }^{15}$ with Wærnhus suggesting that cation vacancies were incorporated into $\mathrm{LaFeO}_{3}$ through Schottky disorder, although this work was unable to identify the type of cation vacancy involved in this disorder and therefore assumed both would be present. ${ }^{16,17}$ The results in Table 7 suggest that $\mathrm{La}_{2} \mathrm{O}_{3}$ partial Schottky and full Schottky defects are likely to form, meaning that vacancies of both cations will be present in the material, although $\mathrm{La}^{3+}$ vacancies may dominate. This finding builds on the conclusions drawn in both earlier studies: $p$-type conductivity is compensated by both $\mathrm{La}^{3+}$ and $\mathrm{Fe}^{3+}$ vacancies formed through partial $\mathrm{La}_{2} \mathrm{O}_{3}$ and full Schottky disorder. We discuss this point in more detail below.

The formation energies of Frenkel and Schottky disorder have also been calculated using interatomic potentials, a more approximate, but computationally cheaper, method. These values are reported in Table 9 along with the DFT $+U$ values for comparison.

Table 9. Energies of Frenkel and Schottky Type Disorder Calculated Using Interatomic Potentials and DFT $+U$

\begin{tabular}{lcc} 
& \multicolumn{2}{c}{ energy defect ${ }^{-1}(\mathrm{eV})$} \\
\cline { 2 - 3 } \multicolumn{1}{c}{ disorder } & interatomic potentials & $\mathrm{DFT}+U$ \\
$\mathrm{La}$ Frenkel & 9.57 & 5.807 \\
$\mathrm{Fe}$ Frenkel & 8.02 & 4.435 \\
$\mathrm{O} 1$ Frenkel & 4.18 & 3.291 \\
$\mathrm{O} 2$ Frenkel & 4.16 & 3.287 \\
full Schottky O1 & 3.52 & 2.207 \\
full Schottky O2 & 3.50 & 2.203 \\
$\mathrm{La}_{2} \mathrm{O}_{3}$ partial Schottky O1 & 3.20 & 2.198 \\
$\mathrm{La}_{2} \mathrm{O}_{3}$ partial Schottky O2 & 3.18 & 2.193 \\
$\mathrm{Fe}_{2} \mathrm{O}_{3}$ partial Schottky O1 & 3.85 & 2.414 \\
$\mathrm{Fe}_{2} \mathrm{O}_{3}$ partial Schottky O2 & 3.84 & 2.409 \\
\hline
\end{tabular}

The formation energies of La and Fe Frenkel disorder differ most, with the values calculated using interatomic potentials being significantly higher in both cases. The majority of the energies calculated for Frenkel and Schottky disorder differ by roughly $1 \mathrm{eV}$. The source of this difference was investigated using each technique to calculate the heat of formation of $\mathrm{LaFeO}_{3}$ in order to compare to the experimental values of $-18.21 \mathrm{eV}^{50} \mathrm{DFT}$ is known to underestimate the heat of formation, whereas interatomic potentials may overestimate the heat of formation.

The heat of formation was calculated with ab initio methods by taking the difference between the cohesive energy of $\mathrm{LaFeO}_{3}$ and the sum of the atoms in their standard state, whereas for interatomic potentials a Born-Haber cycle was used, which led to heats of formation of $-14.70 \mathrm{eV}$ and $-27.02 \mathrm{eV}$ from the 
respective approaches. These results show that although DFT does underestimate the heat of formation and may, as a consequence, underestimate to some degree the vacancy energies, interatomic potentials overestimate it considerably. This overestimation is likely to be the basis of the difference in the defect energies shown in Table 9 and is probably related to the lack of covalency present in the interatomic potential model we used. A degree of covalency has been found in related materials in which it mediates charge transfer and is therefore vital for catalytic processes. $^{51}$

Comparing Frenkel disorder formed from ions with nonzero charge states, interestingly we find lower Frenkel energies for higher charge states of lanthanum, suggesting that formation of interstitials from lattice ions does not result in a change of charge state. However, this is not the case for iron or oxygen. For iron the $\mathrm{Fe}^{2+}$ Frenkel is more stable, which is due to the formation of the displaced interstitial in a $2+$ charge state being stabilized compared to the $\mathrm{Fe}^{3+}$ interstitial. For oxygen, the neutral interstitial is more stable than any of the charged interstitials, suggesting that when an oxygen Frenkel Pair is formed the electrons are left in the vacancy, where they are likely to be stabilized by surrounding iron ions.

Antisite Defects. Another common feature in perovskitetype materials is antisite defects, in which the cation on the Aor $\mathrm{B}$-site replaces the cation in the other site taking the form $\mathrm{AAO}_{3}$ for an A-site ion on the $\mathrm{B}$-site, $\mathrm{BBO}_{3}$ for a B-site ion on the A-site, or $\mathrm{BAO}_{3}$ for an $\mathrm{A}$-site ion on a B-site and a $\mathrm{B}$-site ion on an A-site. The formation of these antisite defects is represented by the following equations:

$$
\begin{aligned}
& \mathrm{La}_{2} \mathrm{O}_{3}+\mathrm{Fe}_{\mathrm{Fe}}^{x} \leftrightarrow \mathrm{La}_{\mathrm{Fe}}^{x}+\mathrm{LaFeO}_{3} \\
& \mathrm{Fe}_{2} \mathrm{O}_{3}+\mathrm{La}_{\mathrm{La}}^{x} \leftrightarrow \mathrm{Fe}_{\mathrm{La}}^{x}+\mathrm{LaFeO}_{3} \\
& \mathrm{La}_{\mathrm{La}}^{x}+\mathrm{Fe}_{\mathrm{Fe}}^{x} \leftrightarrow \mathrm{Fe}_{\mathrm{La}}^{x}+\mathrm{La}_{\mathrm{Fe}}^{x}
\end{aligned}
$$

All three antisite defects were investigated, and the resulting defect formation energies are shown in Table 10. As with the

Table 10. Defect Formation Energies of Antisite Defects Calculated Using DFT $+U$ and Interatomic Potentials

\begin{tabular}{lcc} 
& \multicolumn{2}{c}{ energy defect $^{-1}(\mathrm{eV})$} \\
\cline { 2 - 3 } antisite defect & $\mathrm{DFT}+U$ & interatomic potentials \\
$\mathrm{La}_{\mathrm{Fe}}^{x}$ & 3.010 & 2.85 \\
$\mathrm{Fe}_{\mathrm{La}}^{x}$ & 2.590 & 1.99 \\
$\mathrm{La}_{\mathrm{Fe}}^{x}+\mathrm{Fe}_{\mathrm{La}}^{x}$ & 2.303 & 2.31 \\
\hline
\end{tabular}

case of Frenkel and Schottky disorder, for comparison the defect formation energies of antisite defects have also been calculated using interatomic potentials. For DFT $+U$ calculations, only one energy is reported for each defect, reflecting the fact that the formation energies are independent of the elemental chemical potentials.

The energies calculated for each antisite defect compare well between the two techniques. Although $\mathrm{AAO}_{3}$ has a high formation energy and is therefore not likely to contribute to the defect chemistry of $\mathrm{LaFeO}_{3}, \mathrm{BBO}_{3}$ and $\mathrm{BAO}_{3}$ have formation energies comparable with $\mathrm{La}_{2} \mathrm{O}_{3}$ and full Schottky disorder and therefore may play a role in the chemistry of $\mathrm{LaFeO}_{3}$, particularly at high temperatures.

Investigating the effect of changing the charge state of the ion in the antisite revealed that all charge states, for both ions, have higher formation energies than their neutral counterparts when
$E_{\mathrm{F}}=0$, but as the Fermi energy is increased, lower charge states become more favorable, as can be seen in the following section.

This preferential formation of Schottky-type disorder over antisite disorder is found in other perovskite-based materials, including BSCF in which Schottky disorder causes the material to decompose. ${ }^{48,49}$ Schottky disorder in $\mathrm{LaFeO}_{3}$ has a higher formation energy than in BSCF and, therefore, is not as susceptible to decomposition through this route.

Formation Energies as a Function of Fermi Energy. The formation energies of charged defects depend on the Fermi energy, $E_{\mathrm{F}}$, as seen in eq 2. Therefore, the defect formation energies of charged intrinsic defects, vacancies, interstitials, and antisite defects have been calculated at increasing values of $E_{\mathrm{F}}$, from $0 \mathrm{eV}$, the valence band maximum, to $2.1 \mathrm{eV}$, the conduction band minimum. Figure 6 shows how

O Rich / La \& Fe Poor

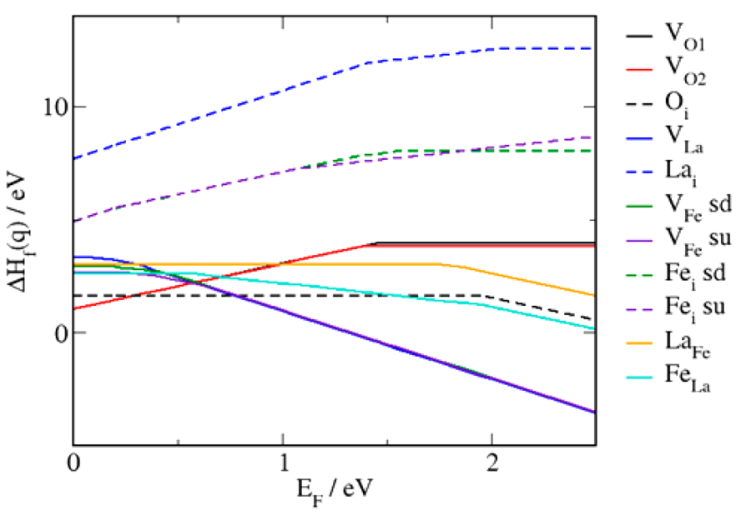

O Poor / La \& Fe Rich

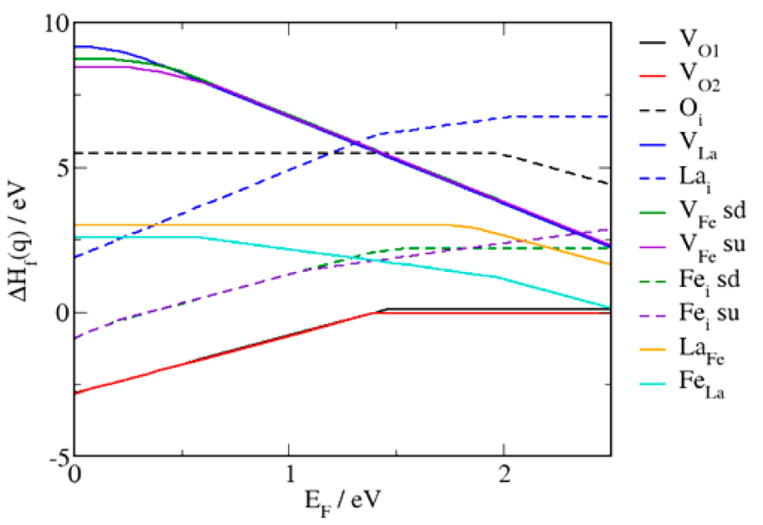

Figure 6. Change in defect energies of intrinsic defects in $\mathrm{LaFeO}_{3}$ with increasing Fermi energy $\left(E_{\mathrm{F}}\right)$ from the VBM $(0)$ to the CBM $(2.1)$, under oxygen rich, lanthanum, and iron poor and oxygen poor, lanthanum, and iron rich conditions.

the formation energy of each defect changes with the Fermi energy within each set of conditions: oxygen rich/lanthanum and iron poor, and oxygen poor/lanthanum and iron rich. In these plots, for each defect only the charge state with the lowest formation energy is shown. The gradient indicates the defect charge state; where the gradient of the lines change, i.e., where the formation energies of two charge states are equal, a thermodynamic transition occurs. For lanthanum and iron defects, $+1,+2$, and +3 charge states have been investigated; for oxygen defects -1 and -2 charge states have been investigated. 
We see from Figure 6 that, for oxygen rich conditions, $\mathrm{O}^{2-}$ vacancies and neutral oxygen interstitials dominate the defect chemistry at low $E_{\mathrm{F}}$ values. However, cation vacancies, both $\mathrm{La}^{3+}$ and $\mathrm{Fe}^{3+}$, become the most favorable defect just before $E_{\mathrm{F}}$ $=1 \mathrm{eV}$. This result further supports the work done by Mizusaki et al. ${ }^{15}$ and Wærnhus et al., ${ }^{16,17}$ by showing that cation vacancies form favorably in oxygen rich conditions.

In oxygen poor conditions oxygen vacancies dominate across all values of Fermi energy, with the $2-$ charge state having the lowest formation energy until $E_{\mathrm{F}}=1.5 \mathrm{eV}$, where the neutral vacancy becomes more favorable. Experimental studies on the conductivity of $\mathrm{LaFeO}_{3}$ under varying oxygen partial pressures show that $n$-type conductivity is observed under low oxygen partial pressures which originates from the formation of oxygen vacancies and a reduction of $\mathrm{Fe}^{3+}$ to $\mathrm{Fe}^{2+} \cdot{ }^{15,16}$ The negative defect formation energy of $\mathrm{O}^{2-}$ vacancies, reported here across all values of Fermi energy, supports the observation of the dominance of $\mathrm{O}^{2-}$ vacancies, and therefore an $n$-type conductivity scheme, under low partial pressures.

The position of the Fermi level in $\mathrm{LaFeO}_{3}$, under oxygen rich and oxygen poor conditions, was calculated using SCFERMI, ${ }^{52,53}$ an in-house developed code, which uses defect formation energies to calculate the concentration of defects and therefore the Fermi level (see Supporting Information). Under oxygen rich conditions, the Fermi level is at $0.87 \mathrm{eV}$, where cation vacancies become the most favorable defect in the system. For oxygen poor conditions, the Fermi level is at 1.55 $\mathrm{eV}$, where the most favorable charge state of oxygen vacancies becomes neutral instead of $2-$.

\section{RELATING OXYGEN CHEMICAL POTENTIAL TO OXYGEN PARTIAL PRESSURE}

In order to compare results of our defect studies to experimental data, it is necessary to relate our theoretical limits of oxygen chemical potential to values of oxygen chemical potential under varying temperature and partial pressure conditions, as these factors can be controlled experimentally. This was done following the method outlined by Reuter and Scheffler. ${ }^{54}$

First, the dependence of oxygen chemical potential on temperature is defined at a constant partial pressure, standard pressure $p^{0}=1 \mathrm{~atm}$ with reference to a zero state; $\mu_{\mathrm{O}}\left(0 \mathrm{~K}, p^{0}\right)=$ $(1 / 2) E_{\mathrm{O}_{2}}^{\text {total }}=0$, as follows:

$$
\begin{aligned}
\mu_{\mathrm{O}}\left(T, p^{0}\right)= & \frac{1}{2}\left[H\left(T, p^{0}, \mathrm{O}_{2}\right)-H\left(0 \mathrm{~K}, p^{0}, \mathrm{O}_{2}\right)\right] \\
& -\frac{1}{2} T\left[S\left(T, p^{0}, \mathrm{O}_{2}\right)-S\left(0 \mathrm{~K}, p^{0}, \mathrm{O}_{2}\right)\right]
\end{aligned}
$$

where $H$ is enthalpy, $T$ is temperature and $S$ is entropy. $\mu_{\mathrm{O}}(T$, $p^{0}$ ) was calculated from 600 to $1500 \mathrm{~K}$, using data from thermochemical tables, ${ }^{55}$ and the results are shown in Table 11.

Table 11. Variation of Oxygen Chemical Potential with Temperature at Standard Oxygen Partial Pressure

$\begin{array}{cccc}\text { temp }(\mathrm{K}) & \mu_{\mathrm{O}}\left(T, p^{0}\right)(\mathrm{eV}) & \text { temp }(\mathrm{K}) & \mu_{\mathrm{O}}\left(T, p^{0}\right)(\mathrm{eV}) \\ 600 & -0.6109 & 1100 & -1.2712 \\ 700 & -0.7295 & 1200 & -1.3998 \\ 800 & -0.8505 & 1300 & -1.5300 \\ 900 & -0.9738 & 1400 & -1.6617 \\ 1000 & -1.0990 & 1500 & -1.7948\end{array}$

Using these values, the chemical potential of oxygen at varying oxygen partial pressures at a given temperature can be calculated via:

$$
\mu_{\mathrm{O}}(T, p)=\mu_{\mathrm{O}}\left(T, p^{0}\right)+\frac{1}{2} k T \ln \left(\frac{p}{p^{0}}\right)
$$

From eq 11, we can calculate oxygen chemical potential values at varying temperatures and pressures, as demonstrated in Tables 12 and 13, respectively. When varying temperature, a

Table 12. Variation in Oxygen Chemical Potential with Temperature at $p_{\mathrm{O} 2}=0.21 \mathrm{~atm}$

$\begin{array}{rccc}\text { temp }(\mathrm{K}) & \mu_{\mathrm{O}}(T, p)(\mathrm{eV}) & \text { temp }(\mathrm{K}) & \mu_{\mathrm{O}}(T, p)(\mathrm{eV}) \\ 600 & -0.6512 & 1100 & -1.3450 \\ 700 & -0.7765 & 1200 & -1.4805 \\ 800 & -0.9043 & 1300 & -1.6175 \\ 900 & -1.0343 & 1400 & -1.7558 \\ 1000 & -1.1663 & 1500 & -1.8956\end{array}$

Table 13. Variation in Oxygen Chemical Potential with Oxygen Partial Pressure at $1000 \mathrm{~K}$

$\begin{array}{cccc}p_{\mathrm{O} 2}(\mathrm{~atm}) & \mu_{\mathrm{O}}(T, p)(\mathrm{eV}) & p_{\mathrm{O} 2}(\mathrm{~atm}) & \mu_{\mathrm{O}}(T, p)(\mathrm{eV}) \\ 1 \times 10^{-9} & -1.9919 & 1 \times 10^{1} & -0.9998 \\ 1 \times 10^{-7} & -1.7935 & 1 \times 10^{3} & -0.8014 \\ 1 \times 10^{-5} & -1.5951 & 1 \times 10^{5} & -0.6030 \\ 1 \times 10^{-3} & -1.3967 & 1 \times 10^{7} & -0.4046 \\ 1 \times 10^{-1} & -1.1983 & 1 \times 10^{9} & -0.2062\end{array}$

partial pressure of 0.21 atm was chosen, as this is a common operating partial pressure for solid oxide fuel cells, ${ }^{7}$ and when varying pressure, a temperature of $1000 \mathrm{~K}$ was chosen, as it falls into the current target operating temperature region for SOFCs: $600-800{ }^{\circ} \mathrm{C}$.

The oxygen chemical potential values used so far in the present study encompass the oxygen chemical potentials in Tables 12 and 13; therefore, defect formation energies under operational conditions will lie between the formation energies calculated in this work under oxygen poor and oxygen rich conditions.

Interestingly, Ritzmann et al. ${ }^{14}$ calculated the free energy of formation of oxygen vacancies, $\Delta G_{\mathrm{f}, \mathrm{vac}}$, at $700{ }^{\circ} \mathrm{C}$, and found that the contribution from entropy is $-0.95 \mathrm{eV}\left(\Delta H_{\mathrm{f} \text {,vac }}\right.$ at 700 ${ }^{\circ} \mathrm{C}=3.99 \mathrm{eV}$ whereas $\left.\Delta G_{\mathrm{f} \text {,vac }}=3.04 \mathrm{eV}\right)$. Our $\mu_{\mathrm{O}}$ at $1000 \mathrm{~K}$, at $1 \mathrm{~atm}$ oxygen partial pressure, is $-1.00 \mathrm{eV}$, suggesting that just taking into account the change in oxygen chemical potential with temperature and pressure provides a good estimation of the entropy contribution to the free energy of vacancy formation.

Stoichiometry of $\mathrm{LaFeO}_{3}$ under Varying Oxygen Partial Pressures. As can be seen in Figure 6, oxygen vacancies will form spontaneously in $\mathrm{LaFeO}_{3}$ under oxygen poor conditions, changing the stoichiometry of the material to $\mathrm{LaFeO}_{3-\delta}$. Jacob and Ranjani ${ }^{56}$ measured the change in stoichiometry of $\mathrm{LaFeO}_{3-\delta}$ with oxygen partial pressure at $1473 \mathrm{~K}$. They represented their results using the following equation,

$$
\ln \delta=-0.1632 \ln \left(\frac{p_{\mathrm{O}_{2}}}{p^{0}}\right)-9.0532
$$


The nonstoichiometry factor, $\delta$, can be evaluated using the following expression,

$$
\delta=N \mathrm{e}^{-\Delta H_{\mathrm{f}}(q) / k T}
$$

where $N$ is the number of species in a formula unit, e.g., 3 for oxygen, $k$ is Boltzmann's constant, and and $\Delta H_{\mathrm{f}}(q)$ is the defect formation energy in charge state $q$. A value for $\mu_{\mathrm{O}}\left(T, p^{0}\right)$ at $1473 \mathrm{~K}$ was interpolated from the values in Table 11 and used to calculated $\mu_{\mathrm{O}}(T, p)$ at a range of partial pressures; Jacob and Ranjani used the range $\ln \left(p_{\mathrm{O} 2} / p^{0}\right)=-30$ to $0 .{ }^{56}$ These oxygen chemical potential values are then used in calculating $\Delta H_{\mathrm{f}}(q)$, thus affecting the value of $\delta$.

Results from the previous section demonstrate that, as well as the oxygen chemical potential changing with oxygen partial pressure, the Fermi level, and therefore Fermi energy, in the material also changes. In Figure 7 we compare results from

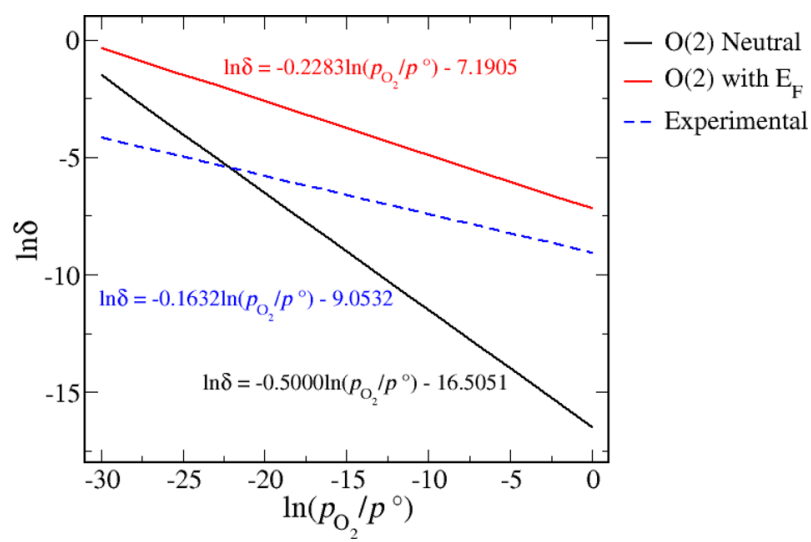

Figure 7. Dependence of the nonstoichiometry factor on oxygen partial pressure with reference to neutral oxygen vacancies and oxygen vacancies calculated at different Fermi energies, from $0.87 \mathrm{eV}$ at $\ln \left(p_{\mathrm{O} 2} / p^{0}\right)=0$ to $1.55 \mathrm{eV}$ at $\ln \left(p_{\mathrm{O} 2} / p^{0}\right)=-30$.

Jacob and Ranjani ${ }^{56}$ to values of nonstoichiometry calculated here using the defect formation energy of neutral oxygen vacancies - which form favorably in oxygen poor conditionsand values of nonstoichiometry calculated using the defect formation energy of oxygen vacancies taking into account the change in Fermi energy-from $0.87 \mathrm{eV}$, in oxygen rich conditions, to $1.55 \mathrm{eV}$ in oxygen poor.

Comparing the fitted trend lines for each set of data, the one fitted to the experimental data of Jacob and Ranjani ${ }^{56}$ reveals that $\delta$ values calculated that take into account the changing Fermi level show a significantly better agreement with the experimental results, validating our estimated Fermi levels and the defect model presented. It is likely that the oxygen rich and oxygen poor conditions, used to calculate the Fermi levels used in the above calculations, correspond to oxygen partial pressures beyond the range used in the experimental nonstoichiometry study, which could be the cause of the difference between our calculated values and the experimental results, and a narrower range may be necessary to improve the agreement between them.

\section{OXYGEN ION MIGRATION}

Oxygen mobility is one of the crucial factors in the use of these materials as electrode materials and is, therefore, investigated in this final section of the study. Due to the inequivalent oxygen sites present in orthorhombic $\mathrm{LaFeO}_{3}$, there are three possible paths for an oxygen ion migrating between two oxygen vacancies: $\mathrm{O} 1$ to $\mathrm{O} 1, \mathrm{O} 1$ to $\mathrm{O} 2$, and $\mathrm{O} 2$ to $\mathrm{O} 2$. All three of these paths were investigated, and their oxygen migration energies were calculated using the procedure stated in Section 2.

The activation energies were calculated with reference to the lowest oxygen vacancy formation energy. Both interatomic potential based methods and DFT $+U$ methods were used, and the results from both methods are shown in Table 14 .

Table 14. Activation Energies of Oxide Ion Migration along the Three Possible Pathways in $\mathrm{LaFeO}_{3}$ Calculated Using Interatomic Potentials and DFT $+U$

\begin{tabular}{ccc} 
& \multicolumn{2}{c}{ activation energy $(\mathrm{eV})$} \\
\cline { 2 - 3 } pathway & interatomic potentials & $\mathrm{DFT}+U$ \\
$\mathrm{O} 2-\mathrm{O} 2$ & 0.50 & 0.58 \\
$\mathrm{O} 1-\mathrm{O} 2$ & 0.63 & 0.66 \\
$\mathrm{O} 1-\mathrm{O} 1$ & 2.06 & 1.87 \\
\hline
\end{tabular}

Starting with the $\mathrm{O} 2-\mathrm{O} 2$ pathway, the activation energy calculated in this work is $0.50 \mathrm{eV}$, which is identical to the activation energy calculated previously by Jones and Islam, ${ }^{10}$ who also used interatomic potentials. However, the method employed here allows for a more complete calculation of the favored position at each site along the path, as multiple points were considered perpendicular to the migration pathway.

The activation energy of the $\mathrm{O} 1-\mathrm{O} 2$ pathway calculated here is $0.63 \mathrm{eV}$, roughly $0.2 \mathrm{eV}$ higher than the value reported by Jones and Islam of $0.44 \mathrm{eV}^{10}$ the possible origin of this discrepancy is discussed later.

Finally, the activation energy of the $\mathrm{O} 1-\mathrm{O} 1$ path is considerably higher than the other two pathways at $2.06 \mathrm{eV}$. This value is supported well by the calculations performed by Jones and Islam who calculated an activation energy of $2.13 \mathrm{eV}$ for this pathway in $\mathrm{LaFeO}_{3}{ }^{10}$ The large magnitude of this activation energy, compared with the activation energies of the other two potential pathways, suggests that migration between two $\mathrm{O} 1$ oxygen sites is unlikely to occur in orthorhombic $\mathrm{LaFeO}_{3}$. The similarity in activation energies calculated for the $\mathrm{O} 1-\mathrm{O} 1$ and $\mathrm{O} 2-\mathrm{O} 2$ pathways between the values calculated in this work and those calculated by Jones and Islam ${ }^{10}$ suggests that the mismatch in values for the $\mathrm{O} 1-\mathrm{O} 2$ pathway is due to differences in the initial oxygen vacancy formation energies. For pathways between the same oxygen sites, these differences cancel out, leading to only the $\mathrm{O} 1-\mathrm{O} 2$ pathway having a significantly different value.

Comparing the values calculated using the different techniques, in general the values agree well with $\mathrm{DFT}+U$, giving a slightly higher value for $\mathrm{O} 2-\mathrm{O} 2$ and $\mathrm{O} 1-\mathrm{O} 2$ but a lower value for $\mathrm{O} 1-\mathrm{O} 1$. The higher activation energies of $\mathrm{O} 2-$ $\mathrm{O} 2$ and $\mathrm{O} 1-\mathrm{O} 2$ brings them closer to the experimental value reported for this material, $0.77 \mathrm{eV},{ }^{57}$ and the similarity in the activation energies of these two pathways suggest that oxide ion migration via both of these routes is likely to take place in this material.

The migration barrier for oxygen ion diffusion in $\mathrm{LaFeO}_{3}$ has been studied previously using $\mathrm{DFT}+U$. Ritzmann et al. ${ }^{14}$ calculated an activation energy of $0.79 \mathrm{eV}$, while Mastrikov et al. ${ }^{45}$ calculated a value of $0.75 \mathrm{eV}$. Both studies used cubic supercells, compared to the orthorhombic cells used in this work, so only one pathway was investigated, which makes comparison with the current study difficult, although suggests 
that migration through orthorhombic $\mathrm{LaFeO}_{3}$ is more favorable than through the cubic structure.

\section{SUMMARY AND CONCLUSIONS}

$\mathrm{DFT}+U$ and static lattice calculations have been used to investigate intrinsic defects in orthorhombic $\mathrm{LaFeO}_{3}$, in order to establish the intrinsic defect structure of the material which will control its response to dopants and influence the material's performance as a SOFC cathode.

We found that lanthanum interstitials form split-interstitials preferentially, whereas iron interstitials are more stable as $\mathrm{Fe}^{2+}$ single interstitials in a tetrahedral site. Frenkel and Schottky type disorder was investigated along with antisite defects. Under oxygen rich conditions, cation vacancies compensate for the holes present in the material. This finding agrees with the $p$ type conductivity that is observed in $\mathrm{LaFeO}_{3}$ under high $\mathrm{O}_{2}$ partial pressures. Under oxygen poor conditions, $\mathrm{O}^{2-}$ vacancies dominate and provide the electrons needed for the $n$-type conductivity that is observed experimentally under low $\mathrm{O}_{2}$ partial pressures.

The nonstoichiometry under varying oxygen partial pressures was investigated and compared to experimental results. An improved agreement with experimental results was found when the change in Fermi level, from $0.87 \mathrm{eV}$ in oxygen rich conditions to $1.55 \mathrm{eV}$ in oxygen poor conditions, was included in the calculation.

The activation energies of all three potential oxide ion pathways in $\mathrm{LaFeO}_{3}$ were calculated with the activation energy of the $\mathrm{O} 1$ to $\mathrm{O} 1$ pathway being significantly higher than the other two. The lowest activation energy calculated was for the pathway between two $\mathrm{O} 2$ sites with the pathway between $\mathrm{O} 1$ and $\mathrm{O} 2$ sites having an activation energy only $0.08 \mathrm{eV}$ higher. Both are within the reasonable range of the experimental activation energy reported in the literature. These low activation energies are expected for a material with good ionic conductivity.

The detailed models for defect structure presented in this paper will provide a valuable guide for optimizing the performance of doped and related materials in solid state electrochemical applications.

\section{ASSOCIATED CONTENT}

\section{(S Supporting Information}

The Supporting Information is available free of charge on the ACS Publications website at DOI: 10.1021/acs.chemmater.6b03048.

Comparison of functionals, analysis of athermal spin state, convergence of supercell size for defect calculations, defect energy as a function of the $U$ parameter, and calculation of self-consistent Fermi level (PDF)

\section{AUTHOR INFORMATION}

\section{Corresponding Author}

*(F.H.T.) E-mail: felicity.taylor.14@ucl.ac.uk.

\section{Author Contributions}

The manuscript was written through contributions of all authors.

\section{Notes}

The authors declare no competing financial interest.

\section{ACKNOWLEDGMENTS}

The authors acknowledge funding from the UCL Chemistry Department. We are grateful to Professor Saiful Islam for useful discussions. J.B. acknowledges funding from the EPSRC project "Energy Materials: Computational Solutions" (EP/K016288/ 1). The authors also acknowledge the use of the UCL Legion and Grace High Performance Computing Facility (Legion@ UCL and Grace@UCL) and the associated support services and the ARCHER supercomputer through the membership of the United Kingdom's HPC Materials Chemistry Consortium, which is funded by EPSRC Grant No. EP/L000202, in the completion of this work.

\section{REFERENCES}

(1) Haile, S. M. Fuel Cell Materials and Components. Acta Mater. 2003, 51, 5981-6000.

(2) Authayanun, S.; Im-orb, K.; Arpornwichanop, A. A Review of the Development of High Temperature Proton Exchange Membrane Fuel Cells. Chin. J. Catal. 2015, 36, 473-483.

(3) Liu, M.; Lynch, M. E.; Blinn, K.; Alamgir, F. M.; Choi, Y. Rational SOFC Material Design: New Advances and Tools. Mater. Today 2011, $14,534-546$.

(4) Aguadero, A.; Fawcett, L.; Taub, S.; Woolley, R.; Wu, K.; Xu, N.; Kilner, J. A.; Skinner, S. J. Materials Development for IntermediateTemperature Solid Oxide Electrochemical Devices. J. Mater. Sci. 2012, 47, 3925-3948.

(5) Jiang, S. P. A Comparison of $\mathrm{O}_{2}$ Reduction Reactions on Porous $(\mathrm{La}, \mathrm{Sr}) \mathrm{MnO}_{3}$ and $(\mathrm{La}, \mathrm{Sr})(\mathrm{Co}, \mathrm{Fe}) \mathrm{O}_{3}$ Electrodes. Solid State Ionics 2002, 146, 1-22.

(6) Shen, F.; Lu, K. Comparative Study of $\mathrm{La}_{0.6} \mathrm{Sr}_{0.4} \mathrm{Co}_{0.2} \mathrm{Fe}_{0.8} \mathrm{O}_{3}$, $\mathrm{Ba}_{0.5} \mathrm{Sr}_{0.5} \mathrm{Co}_{0.2} \mathrm{Fe}_{0.8} \mathrm{O}_{3}$ and $\mathrm{Sm}_{0.5} \mathrm{Sr}_{0.5} \mathrm{Co}_{0.2} \mathrm{Fe}_{0.8} \mathrm{O}_{3}$ Cathodes and the Effect of $\mathrm{Sm}_{0.2} \mathrm{Ce}_{0.8} \mathrm{O}_{2}$ Block Layer in Solid Oxide Fuel Cells. Int. J. Hydrogen Energy 2015, 40, 16457-16465.

(7) Endler-Schuck, C.; Joos, J.; Niedrig, C.; Weber, A.; Ivers-Tiffée, E. The Chemical Oxygen Surface Exchange and Bulk Diffusion Coefficient Determined by Impedance Spectroscopy of Porous $\mathrm{La}_{0.58} \mathrm{Sr}_{0.4} \mathrm{Co}_{0.2} \mathrm{Fe}_{0.8} \mathrm{O}_{3-\delta}$ (LSCF) Cathodes. Solid State Ionics 2015, 269, 67-79.

(8) Develos-Bagarinao, K.; Kishimoto, H.; De Vero, J.; Yamaji, K.; Horita, T. Effect of $\mathrm{La}_{0.6} \mathrm{Sr}_{0.4} \mathrm{Co}_{0.2} \mathrm{Fe}_{0.8} \mathrm{O}_{3-\delta}$ Microstructure on Oxygen Surface Exchange Kinetics. Solid State Ionics 2016, 288, 6-9.

(9) Kuklja, M. M.; Kotomin, E. A.; Merkle, R.; Mastrikov, Y. A.; Maier, J. Combined Theoretical and Experimental Analysis of Processes Determining Cathode Performance in solid oxide fuel cells. Phys. Chem. Chem. Phys. 2013, 15, 5443-5471.

(10) Jones, A.; Islam, M. S. Atomic-Scale Insight into $\mathrm{LaFeO}_{3}$ Perovskite: Defect Nanoclusters and Ion Migration. J. Phys. Chem. C 2008, 112, 4455-4462.

(11) Choithrani, R. Transport Properties of Ferrite Perovskite Material. J. Phys. Sci. Appl. 2012, 2, 245-248.

(12) Cherry, M.; Islam, M. S.; Catlow, C. R. A. Oxygen Ion Migration in Perovskite-Type Oxides. J. Solid State Chem. 1995, 118, 125-132.

(13) Ritzmann, A. M.; Muñoz-García, A. B.; Pavone, M.; Keith, J. A.; Carter, E. A. Ab Initio Evaluation of Oxygen Diffusivity in $\mathrm{LaFeO}_{3}$ : the Role of Lanthanum Vacancies. MRS Commun. 2013, 3, 161-166.

(14) Ritzmann, A. M.; Muñoz-García, A. B.; Pavone, M.; Keith, J. A.; Carter, E. A. Ab Initio DFT $+U$ Analysis of Oxygen Vacancy Formation and Migration in $\mathrm{La}_{1-\mathrm{x}} \mathrm{Sr}_{\mathrm{x}} \mathrm{FeO}_{3-\delta}(\mathrm{x}=0,0.25,0.50)$. Chem. Mater. 2013, 25, 3011-3019.

(15) Mizusaki, J.; Sasamoto, T.; Cannon, W. R.; Bowen, H. K. Electronic Conductivity, Seebeck Coefficient, and Defect Structure of $\mathrm{LaFeO}_{3}$. J. Am. Ceram. Soc. 1982, 65, 363-368.

(16) Wærnhus, I.; Vullum, P. E.; Holmestad, R.; Grande, T.; Wiik, K. Electronic Properties of Polycrystalline $\mathrm{LaFeO}_{3}$. Part I: Experimental Results and the Qualitative Role of Schottky Defects. Solid State Ionics 2005, 176, 2783-2790. 
(17) Wærnhus, I.; Grande, T.; Wiik, K. Electronic Properties of Polycrystalline $\mathrm{LaFeO}_{3}$. Part II: Defect Modelling including Schottky Defects. Solid State Ionics 2005, 176, 2609-2616.

(18) Selbach, S. M.; Tolchard, J. R.; Fossdal, A.; Grande, T. NonLinear Thermal Evolution of the Crystal Structure and Phase Transition of $\mathrm{LaFeO}_{3}$ Investigated by High Temperature X-ray Diffraction. J. Solid State Chem. 2012, 196, 249-254.

(19) Dick, B. G., Jr; Overhauser, A. W. Theory of the Dielectric Constants of Alkali Halide Crystals. Phys. Rev. 1958, 112, 90-103.

(20) Mott, N. F.; Littleton, M. J. Conduction in Polar Crystals. I. Electrolytic Conduction in Solid Salts. Trans. Faraday Soc. 1938, 34, 485-499.

(21) Islam, M. S. Ionic Transport in $\mathrm{ABO}_{3}$ Perovskite Oxides: A Computer Modelling Tour. J. Mater. Chem. 2000, 10, 1027-1038.

(22) Gale, J. D.; Rohl, A. L. The General Utility Lattice Program (GULP). Mol. Simul. 2003, 29, 291-341.

(23) Marezio, M.; Dernier, P. D. The Bond Lengths in $\mathrm{LaFeO}_{3}$. Mater. Res. Bull. 1971, 6, 23-29.

(24) Hohenberg, P.; Kohn, W. Inhomogeneous Electron Gas. Phys. Rev. 1964, 136, B864-B871.

(25) Kohn, W.; Sham, L. J. Self-Consistent Equations Including Exchange and Correlation Effects. Phys. Rev. 1965, 140, A1133A1138.

(26) Dudarev, S. L.; Botton, G. A.; Savrasov, S. Y.; Humphreys, C. J.; Sutton, A. P. Electron-Energy-Loss Spectra and the Structural Stability of Nickel Oxide: An LSDA+U Study. Phys. Rev. B: Condens. Matter Mater. Phys. 1998, 57, 1505-1509.

(27) Blöchl, P. E. Projector Augmented-Wave Method. Phys. Rev. B: Condens. Matter Mater. Phys. 1994, 50, 17953-17979.

(28) Kresse, G.; Hafner, J. Ab initio Molecular Dynamics for OpenShell Transition Metals. Phys. Rev. B: Condens. Matter Mater. Phys. 1993, 48, 13115-13118.

(29) Kresse, G.; Furthmüller, J. Efficient Iterative Schemes for $a b$ initio Total-Energy Calculations using a Plane-Wave Basis Set. Phys. Rev. B: Condens. Matter Mater. Phys. 1996, 54, 11169-11186.

(30) Kresse, G.; Furthmüller, J. Efficiency of ab-initio Total Energy Calculations for Metal and Semiconductors using a Plane-Wave Basis Set. Comput. Mater. Sci. 1996, 6, 15-50.

(31) Perdew, J. P.; Burke, K.; Ernzerhof, M. Generalized Gradient Approximation Made Simple. Phys. Rev. Lett. 1996, 77, 3865-3868.

(32) Perdew, J. P.; Ruzsinszky, A.; Csonka, G. I.; Vydrov, O. A.; Scuseria, G. E.; Constantin, L. A.; Zhou, X.; Burke, K. Restoring the Density-Gradient Expansion for Exchange in Solids and Surfaces. Phys. Rev. Lett. 2008, 100, 136406.

(33) Monkhorst, H. J.; Pack, J. D. Special Points for Brillouin-Zone Integrations. Phys. Rev. B 1976, 13, 5188-5192.

(34) Buckeridge, J.; Taylor, F. H.; Catlow, C. R. A. Efficient and Accurate Approach to Modelling the Microstructure and Defect Properties of $\mathrm{LaCoO}_{3}$. Phys. Rev. B: Condens. Matter Mater. Phys. 2016, 93, 155123.

(35) Arima, T.; Tokura, Y.; Torrance, J. B. Variation of Optical Gaps in Perovskite-type 3d Transition-Metal Oxides. Phys. Rev. B: Condens. Matter Mater. Phys. 1993, 48, 17006-17009.

(36) Koehler, W. C.; Wollan, E. O. Neutron-Diffraction Study of the Magnetic Properties of Perovskite-like Compounds $\mathrm{LaBO}_{3}$. J. Phys. Chem. Solids 1957, 2, 100-106.

(37) Bhargav, K. K.; Ram, S.; Majumder, S. B. Small Polaron Conduction in Lead Modified Lanthanum Ferrite Ceramics. J. Alloys Compd. 2015, 638, 334-343.

(38) Hong, J.; Stroppa, A.; Íñiguez, J.; Picozzi, S.; Vanderbilt, D. Spin-Phonon Coupling Effects in Transitions-Metal Perovskites: DFT $+U$ and Hybrid-Functional Study. Phys. Rev. B: Condens. Matter Mater. Phys. 2012, 85, 054417.

(39) Shein, I. R.; Shein, K. I.; Kozhevnikov, V. L.; Ivanovskiǐ, A. L. Band Structure and the Magnetic and Elastic Properties of $\mathrm{SrFeO}_{3}$ and $\mathrm{LaFeO}_{3}$ Perovskites. Phys. Solid State 2005, 47, 2082-2088.

(40) He, J.; Franchini, C. Screened Hybrid Functional Applied to $3 d^{0}$ $\rightarrow 3 d^{8}$ Transition-Metal Perovskites $\mathrm{LaMO}_{3}(M=\mathrm{Sc}-\mathrm{Cu})$ : Influence of the Exchange Mixing Parameter on the Structural, Electronic, and
Magnetic Properties. Phys. Rev. B: Condens. Matter Mater. Phys. 2012, 86, 235117.

(41) Lany, S.; Zunger, A. Assessment of Correction Methods for the Band-Gap Problem and for the Finite-size Effects in Supercell Defect Calculations: Case studies for $\mathrm{ZnO}$ and GaAs. Phys. Rev. B: Condens. Matter Mater. Phys. 2008, 78, 235104.

(42) Murphy, A. T.; Hine, N. D. M. Anisotropic Charge Screening and Supercell Size Convergence of Defect Formation Energies. Phys. Rev. B: Condens. Matter Mater. Phys. 2013, 87, 094111.

(43) Buckeridge, J.; Scanlon, D. O.; Walsh, A.; Catlow, C. R. A. Automated Procedure to Determine the Thermodynamic Stability of a Material and the Range of Chemical Potentials Necessary for its Formation Relative to Competing Phases and Compounds. Comput. Phys. Commun. 2014, 185, 330-338.

(44) Kroger, A. F. The Chemistry of Imperfect Crystals: Imperfection Chemistry of Crystalline Solids; North-Holland Pub. Co.: Amsterdam, 1974.

(45) Mastrikov, Y. A.; Merkle, R.; Kotomin, E. A.; Kuklja, M. M.; Maier, J. Formation and Migration of Oxygen Vacancies in $\mathrm{La}_{1-\mathrm{X}} \mathrm{Sr}_{\mathrm{x}} \mathrm{Co}_{1-\mathrm{y}} \mathrm{Fe}_{\mathrm{y}} \mathrm{O}_{3-\delta}$ Perovskites: Insight from ab initio Calculations and Comparison with $\mathrm{Ba}_{1-\mathrm{x}} \mathrm{Sr}_{\mathrm{x}} \mathrm{Co}_{1-\mathrm{y}} \mathrm{Fe}_{\mathrm{y}} \mathrm{O}_{3-\delta}$. Phys. Chem. Chem. Phys. 2013, 15, 911-918.

(46) Thomas, B. S.; Marks, N. A.; Begg, B. D. Defects and Threshold Displacement Energies in $\mathrm{SrTiO}_{3}$ Perovskite using Atomistic Computer Simualtions. Nucl. Instrum. Methods Phys. Res., Sect. B 2007, 254, 211-218.

(47) Sokol, A. A.; Walsh, A.; Catlow, C. R. A. Oxygen Interstitial Structures in Close-Packed Metal Oxides. Chem. Phys. Lett. 2010, 492, 44-48.

(48) Kuklja, M. M.; Mastrikov, Y. A.; Jansang, B.; Kotomin, E. A. The Intrinsic Defects, Disordering, and Structural Stability of $\mathrm{Ba}_{\mathrm{x}} \mathrm{Sr}_{1-\mathrm{x}} \mathrm{Co}_{\mathrm{y}} \mathrm{Fe}_{1-\mathrm{y}} \mathrm{O}_{3-\delta}$ Perovskite Solid Solutions. J. Phys. Chem. C 2012, 116, 18605-18611.

(49) Kuklja, M. M.; Mastrikov, Y. A.; Jansang, B.; Kotomin, E. A. First Principles Calculations of $(\mathrm{Ba}, \mathrm{Sr})(\mathrm{Co}, \mathrm{Fe}) \mathrm{O}_{3-\delta}$ Structural Stability. Solid State Ionics 2013, 230, 21-26.

(50) Parida, S. C.; Singh, Z.; Dash, S.; Prasad, R.; Venugopal, V. Thermodynamic Studies on $\mathrm{LaFeO}_{3}(\mathrm{~s})$. J. Alloys Compd. 1998, 280, 94-98.

(51) Akbay, T.; Staykov, A.; Druce, J.; Téllez, H.; Ishihara, T.; Kilner, J. A. The Interaction of Molecular Oxygen on $\mathrm{LaO}$ Terminated Surfaces of $\mathrm{La}_{2} \mathrm{NiO}_{4}$. J. Mater. Chem. A 2016, 4, 13113-13124.

(52) Sourceforge Page for sc-fermi. https://sourceforge.net/projects/ sc-fermi/.

(53) Github Page for sc-fermi. https://github.com/jbuckeridge/scfermi.

(54) Reuter, K.; Scheffler, M. Composition, Structure and Stability of $\mathrm{RuO}_{2}(110)$ as a Function of Oxygen Pressure. Phys. Rev. B: Condens. Matter Mater. Phys. 2001, 65, 035406.

(55) Stull, D. R.; Prophet, H. JANAF Thermochemical Tables, 2nd ed.; NSRDS-NBS (Book 37); National Bureau of Standards U.S.: Washington, DC, 1971.

(56) Jacob, K. T.; Ranjani, R. Thermodynamic Properties of $\mathrm{LaFeO}_{3-\delta}$ and $\mathrm{LaFe}_{12} \mathrm{O}_{19}$. Mater. Sci. Eng., B 2011, 176, 559-566.

(57) Ishigaki, T.; Yamauchi, S.; Mizusaki, J.; Fueki, K.; Naito, H.; Adachi, T. Diffusion of Oxide Ions in $\mathrm{LaFeO}_{3}$ Single Crystal. J. Solid State Chem. 1984, 55, 50-53. 